\title{
Spectroscopy Of Highly Charged Tungsten Relevant To Fusion Plasmas
}

\author{
C. Biedermann, R. Radtke \\ Institut für Physik der Humboldt-Universität zu Berlin, Lehrstuhl Plasmaphysik, \\ Newtonstr 15, 12489 Berlin, and Max-Planck-Institut für Plasmaphysik, \\ EURATOM Association, 17491 Greifswald, Germany
}

\begin{abstract}
The radiation emitted from highly charged tungsten ions has been measured at the Berlin Electron Beam Ion trap using high-resolution x-ray and EUV spectrometers. Highly charged tungsten ions ranging from $\mathrm{Pm}$-like $\mathrm{W}^{13+}$ to $\mathrm{N}$-like $\mathrm{W}^{67+}$ have been produced and excited in EBIT. The line emission originating from electric and magnetic dipole transitions is studied in a wide wavelength range from 0.12 to $80 \mathrm{~nm}$. The results of our measurements are compared with predicted transition wavelengths from atomic structure calculations and observations at the ASDEX Upgrade tokamak. Here we focus on the quasi-continuum emission around $5 \mathrm{~nm}$ from tungsten ions with an open $4 \mathrm{~d}$ shell $\left(\mathrm{In}-\right.$ like $\mathrm{W}^{25+}$ to $\mathrm{Rb}^{-}$-like $\mathrm{W}^{37+}$ ) and the Lshell spectra of ions with configurations close to Ne-like one.
\end{abstract}

Keywords: EBIT, highly charged ions, $\mathrm{x}$-ray and EUV-spectroscopy, recombination, tungsten. PACS: 32.30.Jc, 32.30. Rj

\section{INTRODUCTION}

The Berlin Electron Beam Ion Trap has been established by the Max-PlanckInstitut für Plasmaphysik to generate atomic physics data in support of research in the field of controlled nuclear fusion. In present and future fusion devices, like ASDEX Upgrade, JET or in particular the currently being built ITER, the plasma facing components are and will be protected with high-Z materials, mainly tungsten, due to the advantageous erosion and redeposition properties of this element [1]. Simultaneously the tremendous radiation cooling of the ions from these high-Z materials impose a thread to fusion and demand careful monitoring of the radiation. Measuring the radiation from highly charged ions in the x-ray, extreme ultraviolet and visible spectral ranges provide valuable diagnostics for high temperature plasmas [2]. The spectral information obtained at fusion devices is strongly influenced by the radial profile of electron temperature and density. Additional complications arise since many ionization stages of tungsten are present hampering the identification of individual lines and charge states [3]. The results shown here are achieved with an EBIT device which has the advantage over other plasma sources that a selected ensemble of ions in specific charge states can be produced, stored and excited for spectroscopic investigation. The technique employs stepwise variation of the electron beam energy 
Contribution, invited talk: The $6^{\text {th }}$ International Conference on Atomic and Molecular Data and Their Applications (ICAMDATA)

Beijing, China, October 28-31 2008, to be published as American Institute of Physics Conference Proceedings

Page 2

to scan the ion inventory in the trap across the ionization threshold generating spectra that differ by the contribution of a single charge state.

\section{EXPERIMENT}

EBIT's main component is an electron beam extracted from a heated cathode and accelerated to the three-sectioned drift-tube assembly floating at high potential [4]. Inside the 4-cm-long trap the electron beam is compressed by a strong magnetic field to $70 \mu \mathrm{m}$ diameter producing a confining space-charge potential-well to radially trap ions. Biasing the outer sections of the drift tubes positive with respect to the middle one controls the axial trapping potential. The accelerating drift-tube potential and the space charge trough define the electron beam energy. Atoms are successively ionized by electron impact reaching a maximum abundance of a charge state given by the electron beam energy and the competing recombination, charge exchange and loss processes. For a current of $50 \mathrm{~mA}$ the beam energy spread is about $30 \mathrm{eV} \mathrm{FWHM.}$ Additionally to ionize and confine the highly charged ions, the monoenergetic electron beam can be quickly ramped to excite and probe the trapped ion ensemble. Our target species, tungsten, is evaporated from tungstenhexacarbonyl powder flowing continuously through a differentially pumped gas injector into the trap and being ionized by the electron beam.

The radiation emitted by the highly charged ions confined and excited in EBIT is analyzed by high resolution x-ray and EUV spectrometers [4]. A setup in Bragggeometry with a large-area flat crystal and equipped with a position sensitive detector is used to study $\mathrm{x}$-ray line emission in the wavelength range from 0.04 to $1.6 \mathrm{~nm}$ with a resolution of about $\lambda / \Delta \lambda \approx 1000$. Extreme-ultraviolet emission diagnostics from 3 to $100 \mathrm{~nm}$ was accomplished by means of a $2 \mathrm{~m}$ Schwob-Fraenkel grazing-incidence spectrometer reaching $\lambda / \Delta \lambda \approx 200-4000$. The spectral measurements are calibrated by recording hydrogen- or helium-like reference lines with wavelengths know accurately from standard data base. Auxiliary, broad-band monitoring of the x-radiation between 0.4 and $30 \mathrm{keV}$ energy (in wavelength units: 31 to $0.4 \mathrm{~nm}$ ) is surveyed with a windowless ultra-low energy solid-state germanium detector with a large solid-angle and high detection efficiency.

By increasing the electron beam energy in small steps the abundance of tungsten charge states confined in EBIT is limited to a particular value predetermined by the ionization potential of the specie. Tracking certain spectral features across a range of beam energies and ionization thresholds reveals which lines can be assigned to particular ion charge states. Line identification is supported by comparison with theoretical predictions. 


\section{SPECTROSCOPIC RESULTS}

\section{Band emission at $5 \mathrm{~nm}$}

Fusion relevant spectroscopy of tungsten started at the Oak Ridge tokamak ORMAK in 1977 [5] observing an intense wide, band-like emission structure around 5 $\mathrm{nm}$, which lead to the fear that radiation-loss of high- $\mathrm{Z}$ impurities pose a severe threat to fusion plasmas. Later, further spectroscopic investigation of tungsten was revived

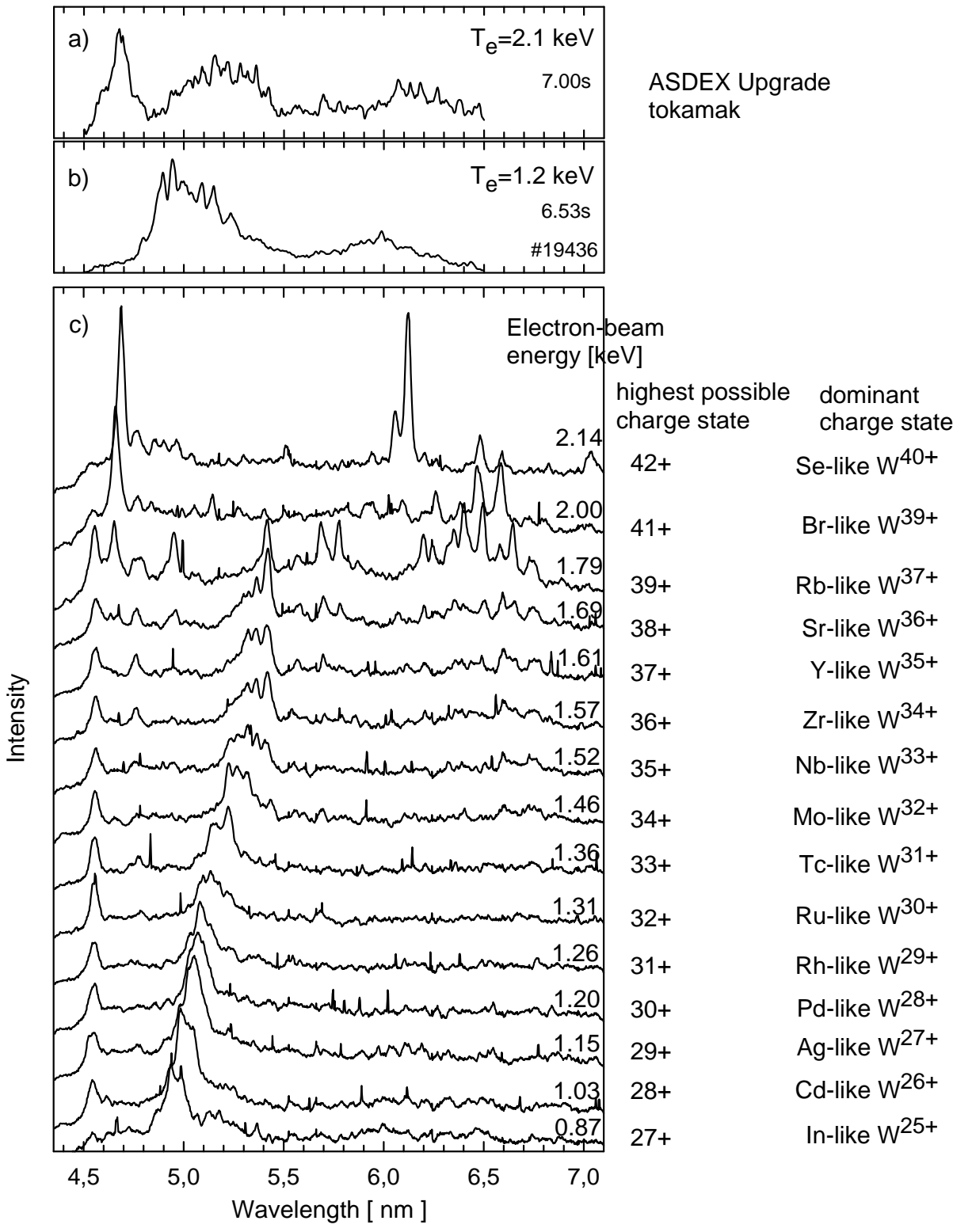

FIGURE 1. Comparison of tungsten spectra from ASDEX Upgrade tokamak and the Berlin EBIT. The tokamak spectra are from discharge \#19436 at 6.53 s corresponding to an electron temperature of $1.2 \mathrm{keV}$ (b) and a hotter plasma at $7.00 \mathrm{~s}$ of $2.1 \mathrm{keV}$ (a) scaled by a factor 3.9. The EBIT spectra (c) are labeled with the electron beam energy in $[\mathrm{keV}]$ and are not scaled relative to each other. Further, the highest possible and the dominant charge state for each spectrum is marked to the right. 
Contribution, invited talk: The $6^{\text {th }}$ International Conference on Atomic and Molecular Data and Their Applications (ICAMDATA) Beijing, China, October 28-31 2008, to be published as American Institute of Physics Conference Proceedings

with studies of the quasi-continuum structure at the ASDEX Upgrade tokamak [6], when tungsten was reconsidered as plasma facing material. Detailed spectroscopic measurements at the Berlin EBIT [4] revealed the fact that several closely spaced lines of many ion states contribute to the structure. For tokamak plasma conditions a range of tungsten ions with open $4 d$ and $4 f$ subshells are present. The large number of coupling possibilities leads to a wealth of levels with similar energies, resulting in a plenitude of lines forming an array. Additional complication arises since tungsten ions in a range of charge states radiate simultaneously in the plasma and the observation integrates the radiation along the line-of-sight. With the technique of increasing the mono-energetic electron beam energy of EBIT in small steps while monitoring the radiation pattern on the experimental side and theoretical effort using HULLAC calculations together with a collisional-radiative model describing the plasma conditions, the wide structure, labelled unresolved transition array, was identified as originating from $4 p-4 d$ and $4 d-4 f$ transitions which slightly shift in wavelength with varying ion state. Figure 1 presents an extract of the observed emission structure between 4.5 and $7 \mathrm{~nm}$ emitted from tungsten ions in charge states $25+$ to $40+$ observed at the Berlin EBIT [4]. In part (c) of Figure 1 the spectra are plotted from bottom to top for increasing electron beam energy, labelled to the right of each graph. The highest possible tungsten charge state given by the ionization potential is limited by the increasing beam energy. The dominant structure of densely spaced lines shifts by about $0.05 \mathrm{~nm}$ per charge state when stepping from In-like tungsten $\mathrm{W}^{25+}$ to $\mathrm{Rb}$-like $\mathrm{W}^{37+}$ with decreasing number of electrons left in the $4 \mathrm{~d}$ shell. The shift in wavelength is also reflected in the ASDEX Upgrade spectra shown in Figure 1(a) and (b) for two different electron temperatures of the plasma, which corresponds to different composition of tungsten ion charge states. For beam energies above $1.7 \mathrm{keV}$ associated to charge states above $38+$ the spectral pattern changes drastically splitting into several separated emission lines. Calculations with the HULLAC package demonstrated that the shift and narrowing of the emission band with respect to statistical-weight modelled spectra can only be explained when considering configuration mixing and applying a collisional-radiative model taking the low plasma density into account. Recently, this information has been confirmed by an independent calculation using the relativistic Dirac-Fock configuration mixing method and assuming an initial ground state approximation [7]. The line observed at $4.5 \mathrm{~nm}$ was identified as $4 p-5 s$ transition.

Many of the ions contributing to the strong electric dipole radiation of the quasicontinuum band observed around $5 \mathrm{~nm}$, also emit magnetic dipole radiation around 70 $\mathrm{nm}$, which appear as isolated lines within a limited wavelength range [8]. The intensity ratio of certain E1/M1 line combinations are strongly dependent on the electron density or the electron temperature, respectively and may serve as valuable plasma diagnostics.

\section{L-shell line spectra}

For much hotter plasmas, as they are envisaged at the future fusion device ITER with an electron temperature of $18 \mathrm{keV}$ for the core region tungsten may be ionized up to $\mathrm{N}$-like $\mathrm{W}^{67+}[1]$. These tungsten ions are ionized to states with an open or emptied 
Contribution, invited talk: The $6^{\text {th }}$ International Conference on Atomic and Molecular Data and Their Applications (ICAMDATA) Beijing, China, October 28-31 2008, to be published as American Institute of Physics Conference Proceedings
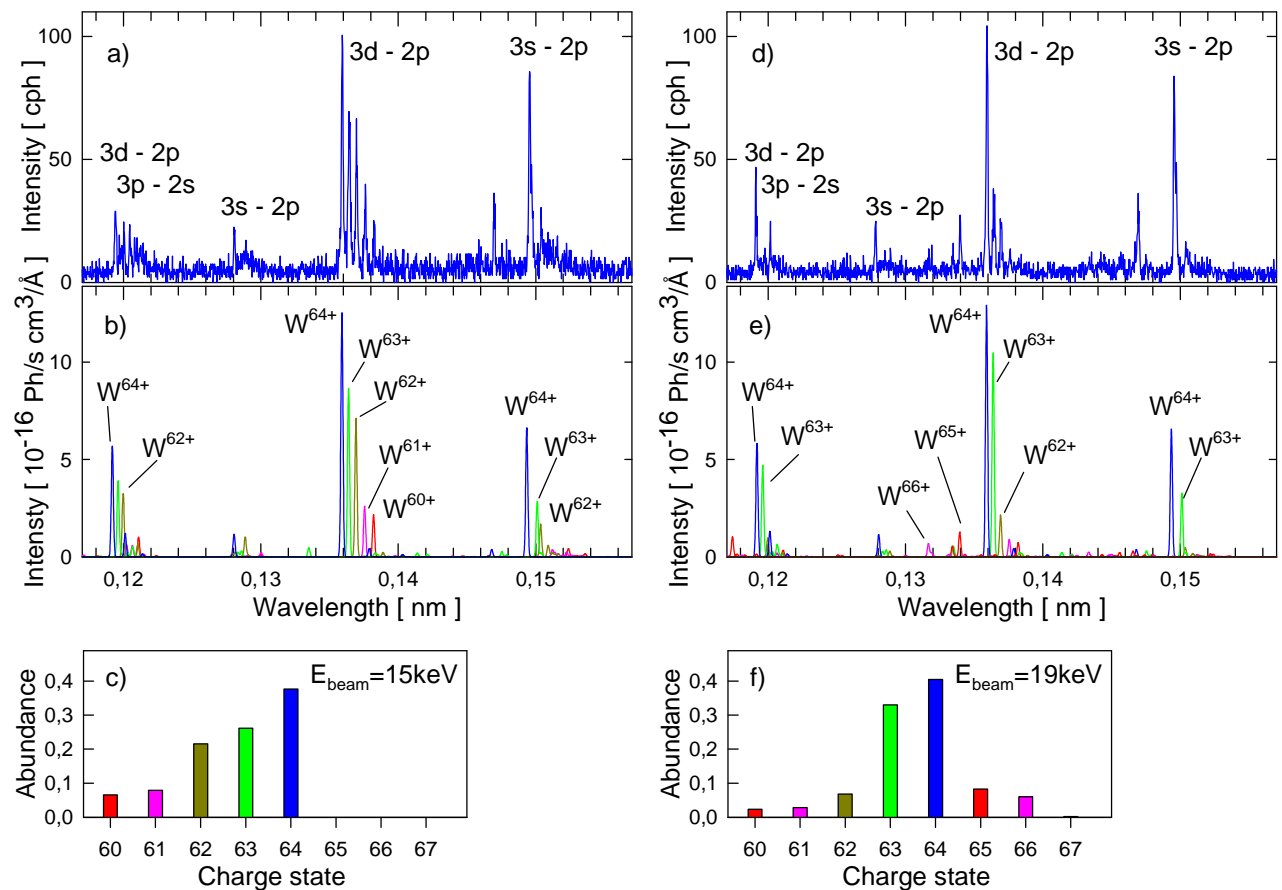

FIGURE 2. X-ray spectra of highly charged tungsten resolved with a Bragg-crystal at two electronbeam energies of EBIT. Left column presents data for $15 \mathrm{keV}$ and the right column for $19 \mathrm{keV}$ beam energy. The second row shows the theoretical prediction by ADAS and Cowan code calculations. The abundance of the tungsten ion charge states is measured with a solid-state detector and plotted in the lower graphs.

$\mathrm{n}=3$ shell and, after excitation, emit strong lines from $\mathrm{n}=3-2$ transitions. This $\mathrm{L}-\mathrm{shell}$ radiation produces lines around $0.13 \mathrm{~nm}$ wavelength. Investigations of the radiation from ions in charge states $60+$ to $67+$ have been started at the Berlin EBIT. The dominant $x$-ray structures emitted from these tungsten ions are analyzed in detail with the flat-crystal spectrometer. Figure 2 shows x-ray spectra acquired at an electron beam energy of $15 \mathrm{keV}$ (Fig. 2(a)) and at $19 \mathrm{keV}$ (Fig. 2(d)). Clearly 4 different groups of lines are observed resulting from $3 s-2 p, 3 d-2 p$ and $3 p-2 s$ transitions and a weaker group at $0.128 \mathrm{~nm}$ from $3 s-2 p$ transitions. Each group is split into a sequence of lines which origin from different ion charge states. The graphs of Fig. 2(a) and (d) are assembled from two spectrometer settings due to the limited wavelength-range accepted by the detector and the narrow rocking curve of the LiF (220) crystal. The lines are identified with the help of computer codes from the ADAS project [9] calculating the atomic structure with the COWAN code [10] and serving as input for a collisional radiative model (corona approximation). The wavelength values have been inspected and adjusted with fully relativistic atomic structure calculations using the GRASP code [11].

The tungsten ion abundance, which serves as an input to the modeling calculations has been extracted from the analysis of a radiative recombination (RR) measurement. In parallel to the high-resolution crystal spectroscopy, the x-rays emitted by the ions trapped and excited in EBIT are registered with a solid-state germanium detector. Since EBIT's electron beam is monoenergetic and has a narrow distribution (FWHM 
Contribution, invited talk: The $6^{\text {th }}$ International Conference on Atomic and Molecular Data and Their Applications (ICAMDATA) Beijing, China, October 28-31 2008, to be published as American Institute of Physics Conference Proceedings

Page 6

$50 \mathrm{eV}$ at $15 \mathrm{keV}$ beam energy) the $\mathrm{x}$-ray signature of the radiative recombination process appears as distinct line in the spectrum. The line energy is given by the binding energy of the capture state, accounting for the $n$ and $l$-state of the recombining ion and the electron beam energy. Fitting the RR-intensity distribution to theoretical well-known RR cross sections and including the electron beam properties it is possible to determine the relevant charge state abundance of the ion inventory in EBIT. Figure 2(c) and (f) present the charge state distributions for the measurement at $15 \mathrm{keV}$ and $19 \mathrm{keV}$ electron beam energy. Below the ionization energy of $\mathrm{W}^{64+}(15.566 \mathrm{keV})$ no radiation of ions with charge states above Ne-like tungsten is observed. At $19 \mathrm{keV}$ electron beam energy up to $\mathrm{N}$-like $\mathrm{W}^{67+}$ can be produced and $\mathrm{x}$-rays associated with radiative recombination are recorded.

With this information on the ion abundance theoretical spectra like Fig. 2(b) and (e) are calculated with the collisional-radiative model to predict intensities and wavelengths using the atomic structure codes. Some of the theoretical lines are spaced very closely. The spectra shown in Fig. 2(b) and (e) are folded with the experimental detector resolution applying a $0.25-\mathrm{pm}-\mathrm{FWHM}$ Gaussian distribution. The spectra demonstrate that each line of a group corresponds to transitions of a particular ion charge state. The predicted intensity relations between different charge state ratios resemble the experimental observation. Each group of lines is headed to lower wavelength values by the dominant Ne-like $\mathrm{W}^{64+}$ line. Ions with lower charge states emit lines that are shifted to larger wavelengths values. These lines from successive ion charge states produce a staircase-like structure. Apart from one line measured with a large intensity at $0.1468 \mathrm{~nm}$, the calculated intensity distribution agrees well with the observed spectrum. Theory predicts a weaker line for an electric quadrupole transition $2 p^{5} 3 p^{3} D_{2}-2 p^{6}{ }^{1} S_{0}$ of $\mathrm{W}^{64+}$ ions at that wavelength.

The information extracted from the detailed spectroscopy at EBIT may help to interpret the spectra observed at the tokamak and design diagnostics of future fusion devices.

\section{REFERENCES}

1. R. Neu, Phys. Scr. T123, 33 (2006).

2. D.P. Stotler, R.E. Bell, K.W. Hill, D.W. Johnson and F.M. Levinton, $5^{\text {th }}$ ICAMDATA, AIP Conference Proceedings 901, 95 (2007).

3. R. Neu, T. Pütterich, R. Dux, A. Pospieszczyk, G. Sergienko, ASDEX Upgrade Team and TEXTOR Team, $5^{\text {th }}$ ICAMDATA, AIP Conference Proceedings 901, 85 (2007).

4. R. Radtke, C. Biedermann, J.L. Schwob, P. Mandelbaum, R. Doron, Phys. Rev. A64, 012720 (2001).

5. R.C. Isler, R.V. Neidigh, R.D. Cowan, Phys. Lett. A63, 295 (1977).

6. K. Assmusen, K.B. Fournier, J.M. Laming, R. Neu, J.F. Seely, R. Dux, W. Engelhardt, J.C. Fuchs, and the ASDEX Upgrade Team, Nucl. Fusion 38, 967 (1998).

7. V. Jonauskas, S. Kučas and R.Karazija, J. Phys. B: At. Mol. Opt. Phys. 40, 2179 (2007).

8. R. Radtke, C. Biedermann, G. Fussmann, J.L. Schwob, P. Mandelbaum, R. Doron, IAEA Atomic and Plasma-Material Interaction Data for Fusion 13, 45 (2007).

9. H.P. Summers, "Atomic data and analysis structure users manual", JET-IR 06, Abingdon: JET Joint Undertaking (1994).

10. R.D. Cowan, "The theory of atomic structure and spectra", Los Alamos Series in Basic and Applied Sciences, Berkeley, CA, University of California Press (1981).

11.I.P. Grant, C.T: Johnson, F.A. Parpia and E.P Plummer, Comput. Phys. Commun. 55, 425 (1989). 